\title{
Attention Differentially Modulates Similar Neuronal Responses Evoked by Varying Contrast and Direction Stimuli in Area MT
}

\author{
Paul S. Khayat, Robert Niebergall, and Julio C. Martinez-Trujillo \\ Cognitive Neurophysiology Laboratory, Department of Physiology, McGill University, Montreal, Quebec H3G 1Y6, Canada
}

The effects of attention on the responses of visual neurons have been described as a scaling or additive modulation independent of stimulus features and contrast, or as a contrast-dependent modulation. We explored these alternatives by recording neuronal responses in macaque area MT to moving stimuli that evoked similar firing rates but varied in contrast and direction. We presented two identical pairs of stimuli, one inside the neurons' receptive field and the other outside, in the opposite hemifield. One stimulus of each pair always had high contrast and moved in the recorded cell's antipreferred direction (AP pattern), while the other (test pattern) could either move in the cell's preferred direction and vary in contrast, or have the same contrast as the AP pattern and vary in direction. For different stimulus pairs evoking similar responses, switching attention between the two AP patterns, or directing attention from a fixation spot to the AP pattern inside or outside the receptive field, produced a stronger suppression of responses to varying contrast pairs, reaching a maximum $(\sim 20 \%)$ at intermediate contrast. For invariable contrast pairs, switching attention from the fixation spot to the AP pattern produced a modulation that ranged from $10 \%$ suppression when the test pattern moved in the cells preferred direction to $14 \%$ enhancement when it moved in a direction $90^{\circ}$ away from that direction. Our results are incompatible with a scaling or additive modulation of MT neurons' response by attention, but support models where spatial and feature-based attention modulate input signals into the area normalization circuit.

\section{Introduction}

It has been established that directing attention to the spatial location, or to nonspatial features of visual stimuli, modulates the responses of visual cortical neurons of primates (Desimone and Duncan, 1995; Treue, 2001; Reynolds and Chelazzi, 2004; Maunsell and Treue, 2006). Some studies have described the modulation caused by spatial attention as a scaling or upward shift of a neuron's response function for attributes such as motion direction (Treue and Martínez Trujillo, 1999), orientation (McAdams and Maunsell, 1999), and contrast (Williford and Maunsell, 2006; Thiele et al., 2009). Conversely, other studies have reported that spatial attention modulates more strongly responses to intermediate-contrast stimuli, producing a shift in the response function along the contrast axis (Reynolds et al., 2000; Martínez-Trujillo and Treue, 2002). The first type of modulation can be described as stimulus/contrast independent, and the second as contrast dependent.

A stimulus/contrast-independent modulation may suggest a mechanism that changes the neuron's response independently of the type (e.g., motion direction, orientation) or strength

Received 0ct. 27, 2009; revised Dec. 14, 2009; accepted Dec. 25, 2009.

This study was supported by a Natural Sciences and Engineering Research Council of Canada (NSERC) fellowship awarded to P.S.K. and Canada Foundation for Innovation, Canadian Institutes of Health Research, and NSERC grants awarded to J.C.M.-T. We thank Dr. E.P. Cook and Dr. C. Pack for providing valuable comments on the manuscript. We thank W. Kucharski and S. Nuara for technical assistance.

Correspondence should be addressed to Julio C. Martinez-Trujillo, Department of Physiology, McGill University, Room 1220, 3655 Prom. Sir. W. Osler, Montreal, QC H3G 1Y6, Canada. E-mail: julio.martinez@mcgill.ca.

DOI:10.1523/JNEUROSCI.5314-09.2010

Copyright $\odot 2010$ the authors $\quad 0270-6474 / 10 / 302188-10 \$ 15.00 / 0$ (luminance/contrast) of the sensory input. Alternatively, a contrastdependent modulation may suggest a process wherein attentional effects are constrained by contrast gain control mechanisms, e.g., attention may modulate the strength of inputs into a neuron, producing an effect similar to changes in stimulus contrast (Reynolds and Heeger, 2009). Some studies have attempted to discriminate between these alternatives by comparing the goodness of fit of model equations to single-cell responses evoked by varying contrast stimuli (Martínez-Trujillo and Treue, 2002; Williford and Maunsell, 2006). They have reported marginal differences between the goodness of fit of the models, with several models explaining most of the variance in the data. Moreover, factors such as differences in the studies' experimental design may have contributed to the reported heterogeneity in results.

In humans, some fMRI studies have described contrastindependent attentional modulation of BOLD signals in visual cortex (Buracas and Boynton, 2007; Murray, 2008), while others have reported contrast-dependent modulations (Li et al., 2008; see also Ekstrom et al., 2009 in the monkey). Behavioral studies have also described both modulation types of psychometric functions (Carrasco, 2006). Thus, the heterogeneity in results also applies to human studies. Recent modeling work has aimed at reconciling the various findings (Ghose, 2009; Lee and Maunsell, 2009; Reynolds and Heeger, 2009). However, experimental data directly clarifying the issue are scarce. Moreover, it remains unknown whether different forms of attention (e.g., spatial and feature based) produce different effects on neuronal responses to varying contrast stimuli. 


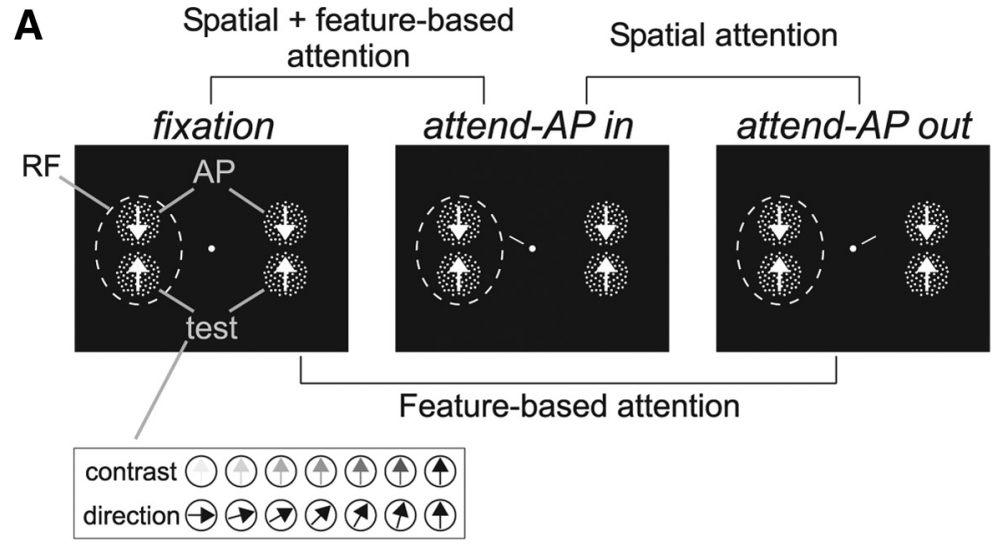

B

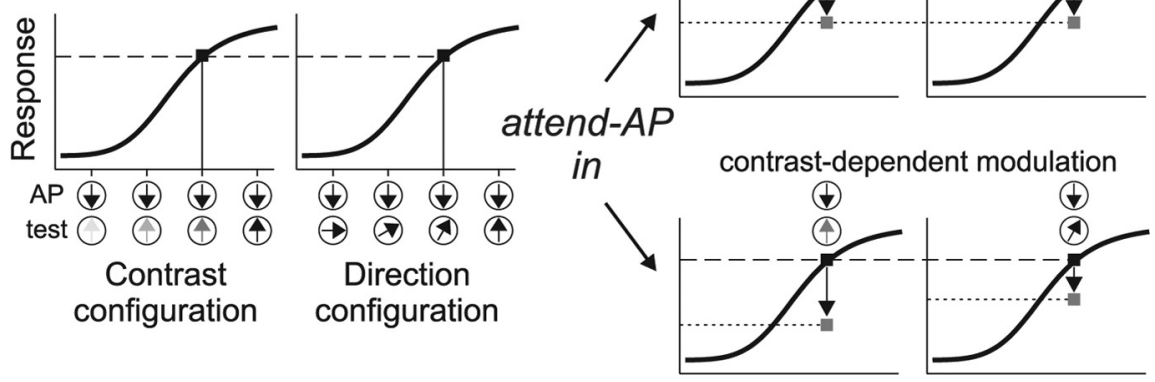

Figure 1. Task design. $\boldsymbol{A}$, Stimulus display in the three behavioral conditions and the different comparisons. Each panel shows two pairs of high-contrast RDPs presented inside and outside the RF (dashed circle), and moving in the neuron's antipreferred (AP pattern) and preferred (test pattern) direction. Insert at the bottom illustrate the different contrasts and motion directions of the test pattern. $\boldsymbol{B}$, The graphs on the left illustrate hypothetical response profiles of an MT direction-selective neuron as a function of changes in contrast or motion direction of the test pattern (illustrated on the abscissa). Black squares mark similar response levels evoked by different stimuli. The graphs on the right illustrate the predictions of the stimulus-independent and contrast-dependent modulation when attention is shifted from the AP pattern outside the RF to a similar pattern inside (arrows and gray squares) (see Materials and Methods for details).

Here, we investigated these issues by comparing the modulation produced by spatial and feature-based attention on the responses of macaque MT neurons to stimuli that varied in direction and contrast but evoked similar responses when unattended. We reasoned that when animals attend to the same location and feature, a stimulus/contrast-independent modulation would produce similar response changes. On the other hand, a contrast-dependent modulation would produce larger changes in responses evoked by stimuli with intermediate contrast. Our data favor the latter explanation, and further show that the modulation produced by spatial, feature-based attention, and their interaction share the same constraints.

\section{Materials and Methods}

Two male macaque monkeys participated in the experiments. Monkey Lu weighed $5.5 \mathrm{~kg}$ and monkey Se $7.2 \mathrm{~kg}$. Standard electrophysiological procedures were used to record neuronal activity in area MT. All surgical procedures for the implant of recording chambers and head holders were conducted under general anesthesia (see Martinez-Trujillo and Treue, 2004). The health of the animals was carefully and periodically monitored by an experienced veterinarian, and the animals were provided with behavioral enrichment protocols when returning to their home cages. All procedures complied with the Canadian Council of Animal Care guidelines and were approved by the McGill animal care committee.

Behavioral task. The animal was required to keep fixation within a $1.5^{\circ}$ window centered on a small spot $\left(0.1\right.$ degree $\left.^{2}\right)$, and then initiate a trial by pressing a button. After $470 \mathrm{~ms}$, two pairs of random dot patterns (RDPs) appeared, one located inside the receptive field (RF) of the recorded MT neuron, and the other located outside, in the opposite hemifield (Fig. 1A). Each pair consisted of a high-contrast RDP moving in the neuron's antipreferred direction (AP pattern) and a second RDP (test pattern) that (1) moved in the neuron's preferred direction but could have, from trialto-trial, different contrast levels (contrast configuration), and (2) had the same contrast as the AP pattern but could move, from trial-to-trial, in different directions (direction configuration) (Fig. $1 \mathrm{~A}$, inset).

We tested three different task conditions. In the "fixation" condition, the animal had to detect a subtle luminance change in the fixation spot, which occurred at a random time between 1010 and $3250 \mathrm{~ms}$ after stimulus onset. In the two other conditions, a small line $\left(1^{\circ}\right.$ length) appeared next to the fixation spot 350 $\mathrm{ms}$ after stimulus onset. This cue-line pointed toward one of the AP patterns, thereby instructing the monkey to direct attention either inside ("attend-AP in" condition) (Fig. $1 \mathrm{~A}$, middle) or outside ("attend-AP out" condition) (Fig. $1 A$, right) the RF. The target underwent a brief direction change 660-2900 ms after cue onset $\left(23^{\circ}\right.$ during $\left.100 \mathrm{~ms}\right)$. The animal had to release the button within a response time window of $150-500 \mathrm{~ms}$ after the change to receive a juice reward. The direction change intensity was chosen in such a way that the animals' performance in most sessions was above $75 \%$ of correct detections.

By comparing responses in the different conditions, we could isolate the effects of spatial and feature-based attention and their combination (Fig. 1A). When the animal switches attention between the AP pattern outside the $\mathrm{RF}$ to the other identical AP pattern inside, only the allocation of spatial attention changes since the attended feature remains the same. When the animal switches attention between the fixation spot and the AP pattern outside the RF, spatial attention remains outside the RF. However, in the feature domain, it is directed from a condition where no motion feature is attended ("fixation") to a condition where the neuron's antipreferred direction is attended. This manipulation has been previously used to isolate feature-based attentional effects in MT neurons (Martinez-Trujillo and Treue, 2004). Finally, switching attention from the fixation spot to the AP pattern inside the RF combines the effect of directing attention into the RF (spatial attention) with the effect of directing attention to the antipreferred direction (feature-based attention).

To guarantee that during the attended conditions the monkey focused attention on the target, the uncued AP pattern located in the opposite visual hemifield changed direction in half of the trials. The monkey had to ignore this distractor change and wait until the target changed. Trials in which the monkey responded to the distractor change or broke fixation were terminated without reward. The different trial types were presented in random sequence, and both animals performed between 6 and 15 trials per stimulus type in each behavioral condition.

Stimuli. The stimuli were back-projected on a screen by a video projector (NEC WT610, $1024 \times 768$ pixels resolution at $85 \mathrm{~Hz}$ ). We used a viewing distance of $57 \mathrm{~cm}$. The RDPs were generated by plotting bright dots $\left(\right.$ dot size $=0.01$ degree $\left.^{2}\right)$ at a density of 4 dots per degree ${ }^{2}$ within a circular stationary virtual aperture on a dark background (luminance $=$ $0.02 \mathrm{~cd} / \mathrm{m}^{2}$ ). Dots moved with $100 \%$ coherence at the preferred speed of the neurons. When they crossed the aperture's border they were replot- 
ted at the opposite side. The size of the RDP (1.3- $3^{\circ}$ diameter) was chosen so that two RDPs fitted inside the boundaries of the classical RF excitatory region.

Stimulus contrast was quantified as the SD of local luminance values (Moulden et al., 1990), and computed as previously described for similar configurations of RDPs (MartínezTrujillo and Treue, 2002). The values were expressed in percentage of the highest contrast value (i.e., AP-pattern contrast $=100 \%$ ). In the contrast configuration, we used different contrast levels of the test pattern obtained by manipulating the luminance of this pattern's dots while keeping constant the background and the AP-pattern luminance. The contrast levels of the test pattern relative to the AP pattern were $0.02,0.1,0.3,0.7,1.5,14$, and $100 \%$. In the direction configuration, we used seven different motion directions of the test pattern (departing from the recorded neuron's preferred direction in steps of $15^{\circ}$ until $90^{\circ}$ away). In this configuration, the contrast of the test pattern was always $100 \%$ (Fig. $1 A$ ).

Recordings and data analysis. Transdural penetrations were made with guide tubes through a chamber implanted on top of a craniotomy of the parietal bone and providing access to area MT. Spikes were recorded using extracellular tungsten electrodes (1-2 $\mathrm{M} \Omega$ at 1 $\mathrm{kHz}, \mathrm{FHC}$ ), and a Plexon data acquisition system (Plexon). The electrode signal was amplified and filtered before being digitized at $40 \mathrm{kHz}$. Single unit activity was isolated using a window discriminator. An interactive stimulus presentation program was used to qualitatively assess the location and size of the neurons' RF, as well as their preferred direction and speed. Cells were determined to be MT units according to their response properties (i.e., direction selectivity and RF position and size), as well as by the position of the electrode relative to the superior temporal sulcus, as localized through MRI scans (Fig. 2). During the recordings, an infrared eye-tracking device (EyeLink) was used to monitor eye position at a sampling frequency of $200 \mathrm{~Hz}$.

The neuron's response was determined by averaging the firing rate across trials within a $600 \mathrm{~ms}$ period that started $150 \mathrm{~ms}$ after cue onset and did not include the stimulus change. We measured neuronal activity during the sustained response period to isolate the modulation produced by endogenous (voluntary) attention, i.e., after the cue was provided to the animals and they directed attention to the target stimulus (Khayat et al., 2006; Busse et al., 2008). This avoids including in our analysis period potential effects of exogenous attention produced by the stimulus/ motion onset transient (Treue and Martínez Trujillo, 1999), as well as underestimates of the firing rate during the early phase of the trial produced by the variability in response latency associated with changes in the test stimulus contrast (Reynolds and Desimone, 2003; Lee et al., 2007). Cells were included in the analyses if at least six correctly performed trials per stimulus and condition were available (median $=12$ trials; first and third quartiles equal 8 and 14 trials, respectively). A total of 101 units (65 in monkey Se and 36 in monkey Lu) were included in the analyses, and were recorded during 96 sessions (in 5 sessions two units were simultaneously isolated from the same electrode).

Population responses were obtained by first, normalizing each cell response to the response evoked by the optimal stimulus during the "fixation" condition, and then, pooling across neurons. The optimal stimulus was defined as the combination of the AP pattern and a full $(100 \%)$ contrast test pattern moving in the cell's preferred direction. To quantify the strength of attentional effects between responses in the different conditions, we computed a modulation index (MI) using the following equation: $\left(R_{\mathrm{c} 1}-R_{\mathrm{c} 2}\right) /\left(R_{\mathrm{c} 1}+R_{\mathrm{c} 2}\right)$, where $R_{\mathrm{c} 1}$ and $R_{\mathrm{c} 2}$ are the responses in the two conditions to be compared (Treue and Martínez Trujillo, 1999). To extract, for each neuron, equated (similar) responses between stimuli in the direction and contrast configuration, the response evoked by each stimulus pair (in a $600 \mathrm{~ms}$ analysis period) was first normalized to the response evoked by the optimal stimulus pair, which was common to both configurations. We then equated the responses in the contrast configuration during either the "attend-AP out" or "fixation" condition to those in the same condition of the direction configuration, i.e., by finding corresponding response levels between the two configurations. This allowed obtaining equated normalized responses and computing the corresponding MIs for each response level. ANOVA and $t$ tests were used to test for significant effects in the MIs distribution.

We also determined the modulation in task performance by computing a performance modulation index, perfMI $=\left(\operatorname{Perf}_{\mathrm{c} 1}-\operatorname{Perf}_{\mathrm{c} 2}\right) /\left(\operatorname{Perf}_{\mathrm{c} 1}+\right.$ $\operatorname{Perf}_{\mathrm{c} 2}$ ), where $\operatorname{Perf}_{\mathrm{c} 1}$ and $\operatorname{Perf}_{\mathrm{c} 2}$ are the hit rates (proportion of correct direction change detections) corresponding to the two conditions to be compared. Ninety-five percent confidence intervals for each perfMI were computed using a bootstrap procedure (10,000 resampling). Comparisons between perfMIs were conducted using nonparametric KruskalWallis ANOVA and sign tests.

\section{Results}

Attentional modulation as a function of response level

The main idea behind our experimental design is illustrated in Figure $1 B$. The left panels display hypothetical response profiles (i.e., with attention directed outside the neuron's RF) of an MT direction-selective unit to two different stimulus configurations resembling the ones used in our experiment, each consisting of two moving RDPs presented inside the cell's RF. The changes in the test-pattern contrast or direction produce similar response profiles. However, resulting from different stimuli. For example, the AP pattern paired with a low-contrast test pattern moving in the cell's preferred direction will evoke the same response as when paired with a high-contrast test pattern moving in a direction different from preferred.

This experimental situation leads to clear predictions on the effects of attention on neuronal responses. If attention produces a response modulation independent of the stimulus type (direction 


\section{A \\ Single-unit example}

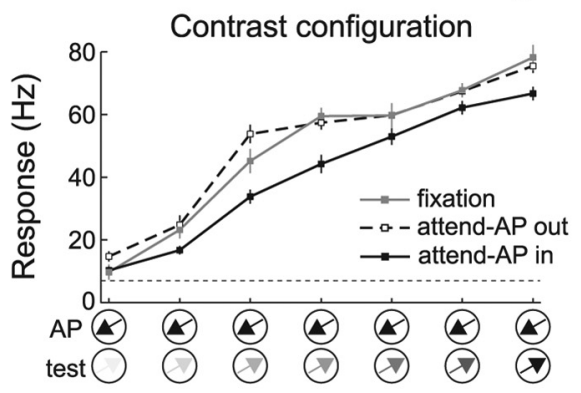

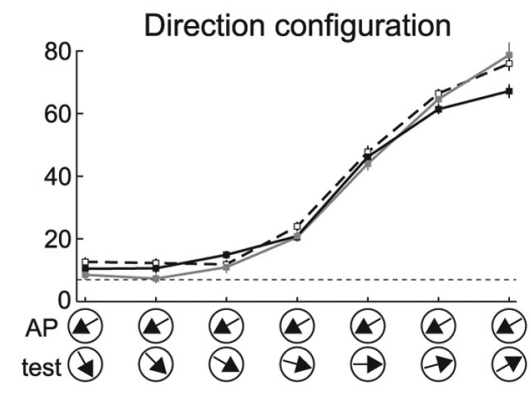

B

attend-AP in vs. attend-AP out

C
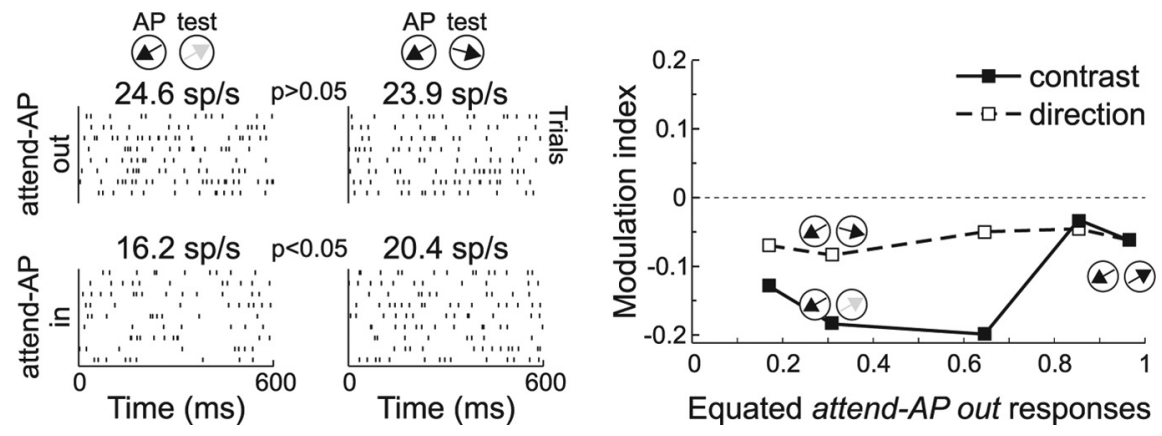

Figure 3. Attentional modulation of equated responses: single-unit example. $\boldsymbol{A}$, Average responses to different combinations of AP and test-pattern contrasts (left) and directions (right) in the "fixation" (gray), "attend-AP in" (black), and "attend-AP out" (dashed) conditions. The stimuli are illustrated along the abscissa. The dashed horizontal line indicates spontaneous response without stimulus. The neuron's preferred direction was $60^{\circ}$ clockwise from vertical. Error bars, SEM. B, Raster plots of the neuron's activity, and mean firing rates during trials with different stimulus pairs, in the "attend-AP out" (top) and "attend-AP in" (bottom) conditions. The $p$ value indicates the significance level ( $U$ test) of differences between responses evoked by different stimuli. $\boldsymbol{C}, \mathrm{MI}$ across responses equated during the "attend-AP out" trials. Black squares and solid line, MI for responses to stimuli of the contrast configuration. Open squares and dashed line, Ml for responses to stimuli of the direction configuration.

or contrast) but dependent on the response level, when switching attention between the two AP patterns the neuron's response to both stimulus combinations should be modulated by a similar amount (Fig. $1 B$, top right). As an alternative, a contrastdependent modulation predicts that the response change will be larger for the combination with the lower contrast (Fig. $1 B$, bottom right). Note that this design keeps the allocation of attention constant during both, the contrast and the direction configuration trials (at the same location and on the same feature), regardless of changes in the test pattern identity, and without substantially altering task difficulty. It also groups features of two previous studies, one reporting multiplicative changes in response with attention (Treue and Martínez Trujillo, 1999), and the other reporting a contrast-dependent attentional modulation (Martínez-Trujillo and Treue, 2002).

We recorded the responses of $101 \mathrm{MT}$ single neurons to the two stimulus configurations in two macaques during task trials of the three different conditions, "fixation," "attend-AP in," and "attend-AP out" (see Materials and Methods). Figure $3 A$ shows responses of a single-unit to different stimulus pairs of the contrast (left) and direction configuration (right). Responses during "fixation" trials (gray line) were strongest when the test pattern had high contrast and moved in the preferred direction (rightmost data point in each panel), and became weaker as the test pattern decreased contrast, or changed direction away from preferred. Visual inspection of these data suggests that the magnitude of the differences in response between the "attend-AP in" (black) and "attend-AP out" (dashed line) conditions varied between the two stimulus configurations.
We investigated whether directing spatial attention inside the RF produced a similar effect on the same response level evoked by distinct stimulus combinations. Figure $3 B$ shows raster plots of the neuron's response to two different stimulus pairs. Responses during the "attend-AP out" condition were similar when the test pattern had intermediate contrast and moved in the preferred direction (contrast pair, top left raster), and when it had high contrast and moved in a direction $45^{\circ}$ away from preferred (direction pair, top right raster) (response $=24.6 \pm 3.5$ SEM and $23.9 \pm 2.4$ SEM spikes/s for the contrast and direction pairs, respectively; $p>0.05, U$ test). When directing attention to the AP pattern inside the RF, the stimulus-independent modulation predicts that responses to both pairs will be modulated in the same manner. However, we found that in the "attend-AP in" condition (bottom rasters) the response to the contrast pair was significantly suppressed (16.2 \pm 1.4 SEM spikes/s, $p<0.05$, $U$ test), while the response to the direction pair did not significantly change $(20.4 \pm 1.3$ SEM spikes/s, $p>0.05, U$ test).

In the same unit, we normalized responses evoked by all stimulus pairs to the response evoked by the combination of AP pattern and maximal contrast preferred test pattern during "fixation" (black rightmost data point in both panels of Fig. $3 A$ ). We then identified similar response levels during trials of the "attend-AP out" condition that were evoked by different stimulus combinations (equated normalized responses between contrast and direction combinations, see Materials and Methods). To determine the strength of the modulation for each response level, we computed a modulation index $\mathrm{MI}=\left(R_{\text {in }}-R_{\text {out }}\right) /\left(R_{\text {in }}+R_{\text {out }}\right)$, where $R_{\text {in }}$ and $R_{\text {out }}$ are the responses to each stimulus combination in the "attend-AP in" and "attend-AP out" conditions, respectively (Fig. 3C). Across different levels of equated "attend-AP out" responses, attending to the AP stimulus inside the RF suppressed more strongly responses to contrast (black squares) compared to direction (open squares) combinations.

We conducted the same analyses for each recorded neuron $(n=101)$, and then averaged the MIs across units within each equated "attend-AP out" normalized response level (Fig. 4). Overall, spatial attention (Fig. $4 \mathrm{~A}$ ) ("attend-AP in" vs "attend-AP out") produced a stronger modulation in the contrast configuration. The MIs were more negative for responses evoked by contrast (black squares and solid line) relative to direction (open squares and dashed line) stimulus pairs. The stars on the abscissa in each panel indicate the equated response levels with significant differences in MIs between trials of both configurations $(p<$ 0.05 , paired $t$ test).

We further investigated whether feature-based attention and its combination with spatial attention produced a similar modulation. We equated responses during "fixation," and compared them to those of the "attend-AP in" and "attend-AP out" conditions. Note that the "fixation" condition is considered in this context as feature-neutral, since attention is away from a mo- 
tion feature and from the RF (Fig. 1A) (Martinez-Trujillo and Treue, 2004). We found that the MIs for the comparison "attend-AP in" versus "fixation" (spatial + feature-based attention) (Fig. 4C) $[\mathrm{MIs}=$ $\left.\left(R_{\text {in }}-R_{\text {fix }}\right) /\left(R_{\text {in }}+R_{\mathrm{fix}}\right)\right]$, and "attend-AP out" versus "fixation" (feature-based attention) (Fig. $4 E)\left[\mathrm{MIs}=\left(R_{\text {out }}-R_{\text {fix }}\right) /\left(R_{\text {out }}+\right.\right.$ $\left.R_{\text {fix }}\right)$ ] were significantly more negative for stimuli of the contrast configuration $(p<$ 0.05 , paired $t$ test) (Fig. 4C,E, stars).

Overall, these data show that at the level of the population, responses evoked by contrast pairs were more strongly suppressed by spatial attention ("attend-AP in" vs "attend-AP out"), feature-based attention ("attend-AP out" vs "fixation"), and the interaction of both types of attention ("attend-AP in" vs "fixation") than similar responses evoked by direction pairs. The differences in the modulation strength were in some cases as large as $20 \%$ (third and fourth data points from right to left in Fig. $4 C$ ).

One alternative explanation for the effects isolated in Figure 4, $A, C$, and $E$, is that they were caused by variations in the animals' attentional effort, due to differences in task difficulty. We consider this possibility very unlikely since in general, increases in attentional effort increase the attentional modulation (Spitzer et al., 1988; Ghose and Maunsell, 2002; Boudreau et al., 2006). In our scenario, it only makes sense to hypothesize that low-contrast test patterns were "easier to filter out" relative to the high-contrast direction stimuli leading to less attentional effort. Our results show the largest modulation for the lower contrast stimuli, which is exactly the opposite as predicted by this hypothesis. Nevertheless, we investigated this possibility by computing the animals' performance for trials that contributed to each equated response level, and expressed it as perfMI (see Materials and Methods). Changes in attentional effort should be reflected in the animals' performance, and if they caused the isolated differences in response modulation between conditions and stimulus configurations, the perfMIs should be correlated with the corresponding attentional MIs.

Figure $4 B$ shows average perfMIs for trials corresponding to the response data shown in Figure $4 A$, and for the contrast and direction configuration. The perfMIs were not different from zero ( $p>0.2$, sign test for each stimulus pair), and were similar across all response levels and stimulus configurations ( $p>0.9$, Kruskal-Wallis ANOVA), indicating that the animals performed similarly regardless of the attended target location, and of the test-pattern contrast or direction. This suggests that the differences in response between the different conditions and stimulus configurations were not due to variations in the animals' attentional effort.

Figure $4 D$ shows the perfMI corresponding to the response data in Figure $4 C$. The perfMIs were negative at each response response level.
A attend-AP in vs. attend-AP out

B

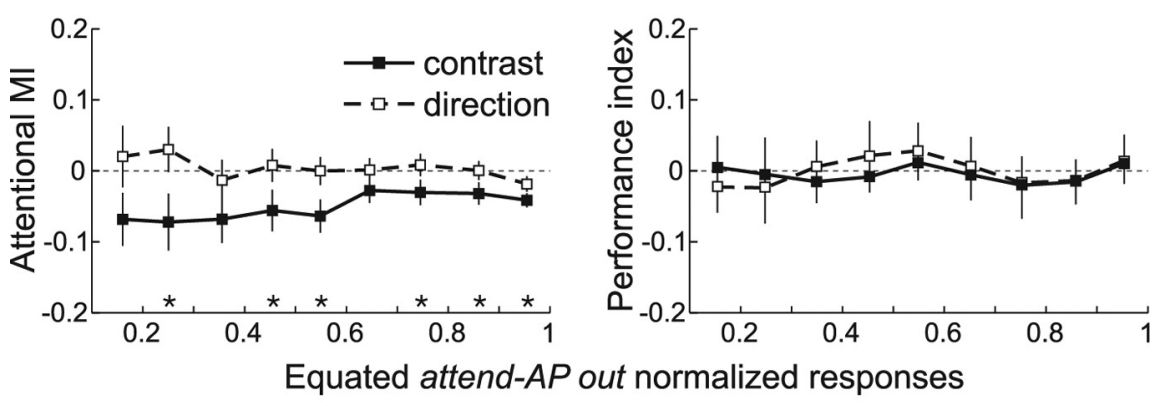

C attend-AP in vs. fixation D

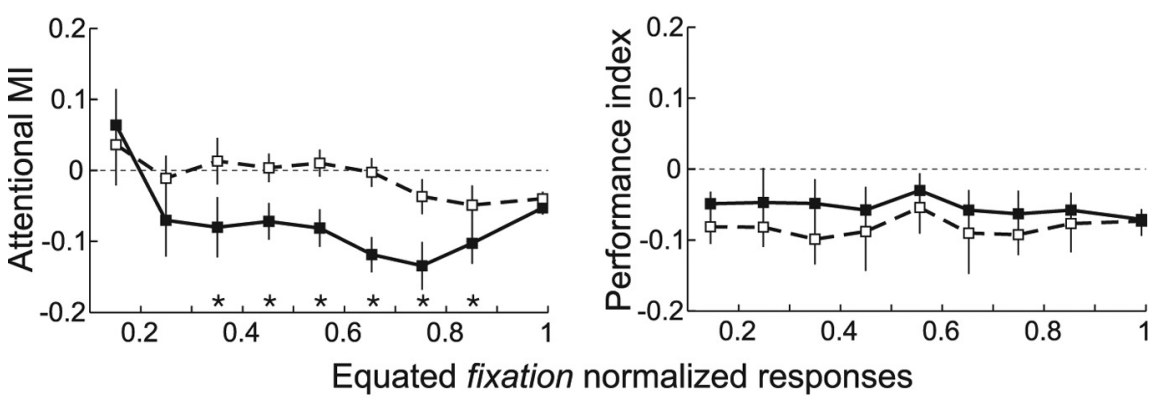

E attend-AP out vs. fixation $\mathbf{F}$

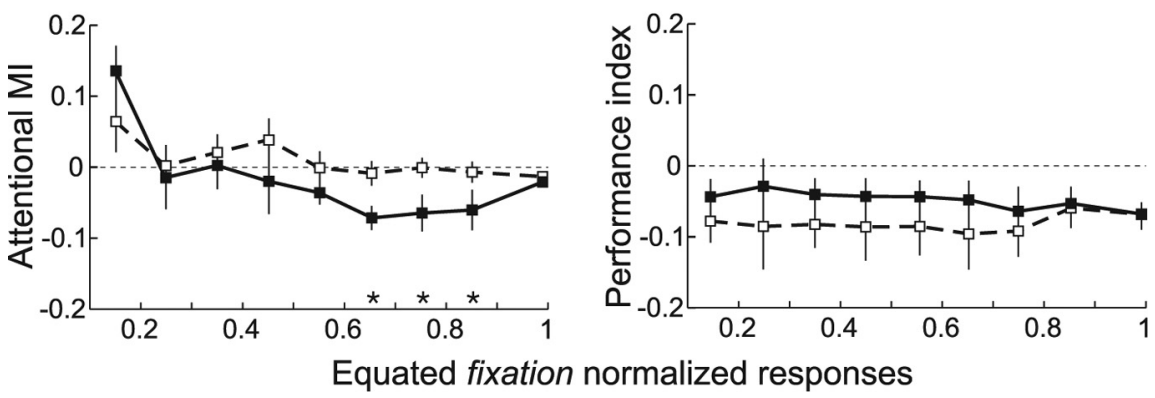

Figure 4. Attentional modulation of equated responses: population analysis. A, Averaged population MIs between "attend-AP in" and "attend-AP out" trials across response levels equated during the "attend-AP out" condition. Error bars, SEM. Stars denote equated response levels with significant differences in MI between trials of the contrast (black squares) and direction (open umber of cells contributing to each response level also varied. B, Averaged performance indices (perfMI) between "attend-AP in" "attend-AP out" trials that contributed to each equated "attend-AP out" response level. Error bars, $95 \%$ confidence intervals igh a bootstrap procedure). $\boldsymbol{C}, \boldsymbol{E}$, Averaged population Mls between "attend-AP in" and "fixation" $(\boldsymbol{C})$ and between "attend-AP out" and "fixation" trials $(\boldsymbol{E})$ across responses equated during the "fixation" condition. $\boldsymbol{D}, \boldsymbol{F}$, Averaged performance indices between "fixation" trials and "attend-AP in" (D) or "attend-AP out" trials $(\boldsymbol{F})$ that contributed to each equated "fixation"

level ( $p<0.05$, sign test), indicating that performance during "fixation" was higher than during "attend-AP in" trials. However, we did not find significant differences in the perfMI across response levels, and between trials of the contrast and direction configurations ( $p>0.2$, Kruskal-Wallis ANOVA). Additionally, perfMIs between trials of the "fixation" and "attend-AP out" conditions were very similar to the ones between trials of the "fixation" and "attend-AP in" conditions (Fig. 4, compare D, F) $(p>0.3$, Kruskal-Wallis ANOVA). However, the response modulation between the conditions and the differences between contrast and direction pairs were larger in the latter case, reaching significance for almost all equated response levels but the two lowest and the highest (Fig. 4, compare C, E). We hypothesize that these larger effects are due to the combined effect of spatial and feature-based attention (attending to the antipreferred direction and inside the RF) in Figure $4 C$ compared to the smaller effect of feature- 

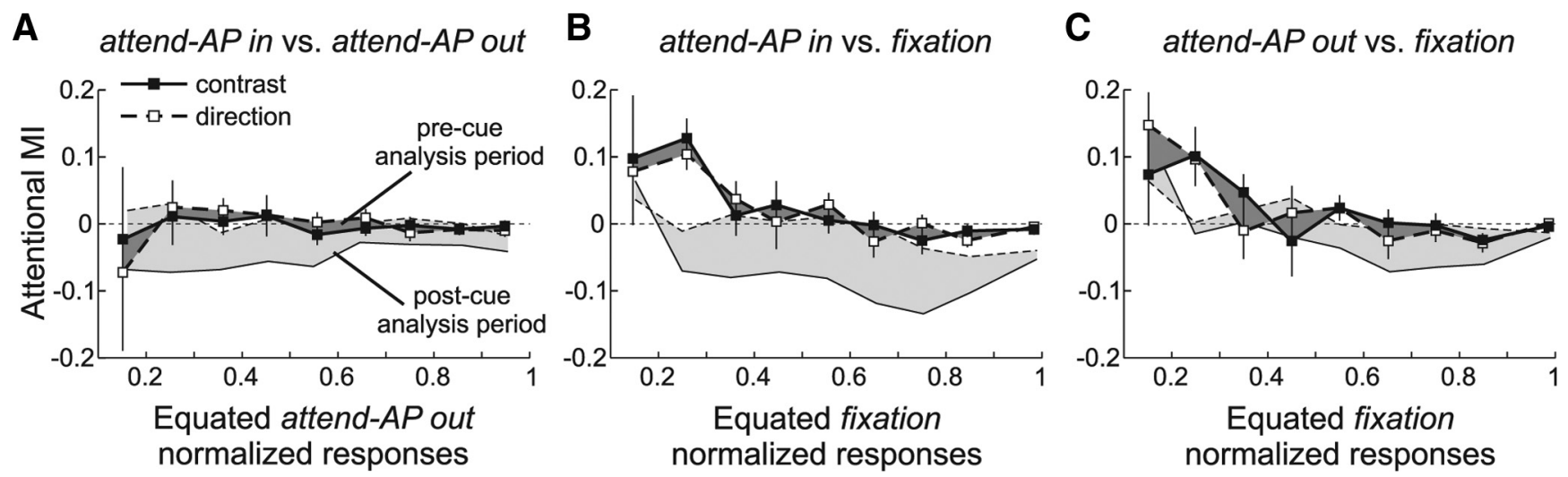

Figure 5. Control data. $\boldsymbol{A}-\boldsymbol{C}$, Mls of the different comparisons for "attend-AP out" ( $\boldsymbol{A}$ ) and "fixation" ( $\boldsymbol{B}, \boldsymbol{C}$ ) responses equated during an analysis period before the attentional cue onset. The dark area represents the MI difference between the two stimulus configurations, computed during the precue analysis period. For comparison, the data from the postcue analysis period, as in Figure 4 , are also shown (light area). The same conventions are used as in Figure 4.

based attention (attending to the antipreferred direction away from the RF) in Figure $4 E$ (see Treue and Martínez Trujillo, 1999). These results strongly argue against the role of attentional effort in the differences in response modulation between the contrast and direction configurations.

We conducted an additional control to test whether our results were indeed caused by changes in the animals' allocation of attention following the instructions provided by the cue. We recomputed the MI across equated responses during a $400 \mathrm{~ms}$ time period previous to the cue onset. For all three comparisons, the differences in MIs between the contrast and direction data disappeared (Fig. 5, dark area) ( $p>0.05$, paired $t$ test at each response level), indicating that effects isolated in the previous analysis were tightly coupled to the instructions provided by the cue.

All together, our findings argue against a stimulus-independent modulation of responses by attention. They rather show that contrast and direction pairs were differentially modulated by the three types of attention.

\section{Attentional modulation as a function of the test-pattern contrast}

Previous studies have reported that the magnitude of attentional modulation is stronger for neuronal responses evoked by stimuli with intermediate compared to low or high contrast (Reynolds et al., 2000; Martínez-Trujillo and Treue, 2002). The previous analysis could not clearly replicate this result since it may have pooled, within a given response level, similar responses evoked by different stimulus pairs of the contrast configuration. To address this issue, we computed population responses $(n=101)$ in the different conditions for each contrast level of the test pattern.

We normalized each neuron's response in the contrast configuration to the response evoked by the maximal contrast stimulus pair (AP + preferred direction test pattern) during "fixation" trials, and then averaged responses to identical stimulus pairs in each condition across units (Fig. 6A). Responses in the "fixation" condition (gray) decreased as the contrast of the test pattern decreased. Attending to the AP pattern yielded an overall response suppression that varied as a function of contrast (larger along the slope of the contrast response function and smaller on the extremes). This suppression was stronger in the "attend-AP in" (black) relative to the "attend-AP out" condition (dashed line). We quantified these observations by computing MIs for the different comparisons (Fig. 1A) for each individual neuron and stimulus combination, and then averaging across indices.
Figure $6 \mathrm{~B}$ shows the average MIs for the comparison "attend-AP in" versus "attend-AP out" conditions. For all stimulus combinations the MIs were significantly lower than zero (open squares, $p<0.05, t$ test), indicating that responses were suppressed in the former condition. Responses during trials with the highestcontrast test pattern were suppressed by $5.5 \%$ (rightmost data point, $\mathrm{MI}=-0.027, p<0.02$, $t$ test), and reached $15 \%$ suppression as the contrast of the test pattern decreased $(\mathrm{MI}=-0.083$, $p<0.0001, t$ test; second data point from left to right). A paired comparison between MIs for high- and intermediate-contrast stimuli yielded significance ( $p<0.005$, paired $t$ test). These results indicate that the response modulation produced by shifting spatial attention into the RF was stronger for intermediatecontrast stimuli, and decreased for the lowest- and highestcontrast stimuli. These results are similar to the ones reported in previous studies of attention (Reynolds et al., 2000; MartínezTrujillo and Treue, 2002).

To examine whether the changes in the strength of attentional modulation across the various contrasts was due to changes in task difficulty, we computed the perfMI between trials of the "attend-AP in" and "attend-AP out" conditions (Fig. 6B, dashed line). Performance in both conditions was similar (perfMI not different from zero, $p>0.3$, sign test for each contrast pair) and did not change across stimuli $(p>0.8$, Kruskal-Wallis ANOVA). This result demonstrates that all trial types in both tasks were correspondingly difficult, suggesting that this variable could not have caused the observed pattern of attentional modulation. This conclusion also holds against the improbable argument that our performance measurements may not reflect attentional effort since the trials that theoretically would lead to the highest and lowest efforts $(100 \%$ vs $\sim 0 \%$ contrast, rightmost and leftmost data points, respectively) led to a similar attentional modulation, i.e., the magnitude of the attentional modulation corresponding to these trials was weaker and more similar than the one corresponding to trials with intermediate contrast $(p<0.005$, paired $t$ test $)$.

We further compared the responses between the "attend-AP in" and "fixation" conditions (Fig. 6C). Except for the lowestcontrast combination (black square), the MI was significantly below zero (open squares, $p<10^{-6}, t$ test), indicating that responses were suppressed in the "attend-AP in" condition relative to "fixation." Responses during trials with the highest-contrast stimulus combination were suppressed by $10 \%$ (rightmost data point, $\mathrm{MI}=-0.051, p<10^{-6}, t$ test $)$, and reached $23 \%$ suppres- 
A Contrast configuration

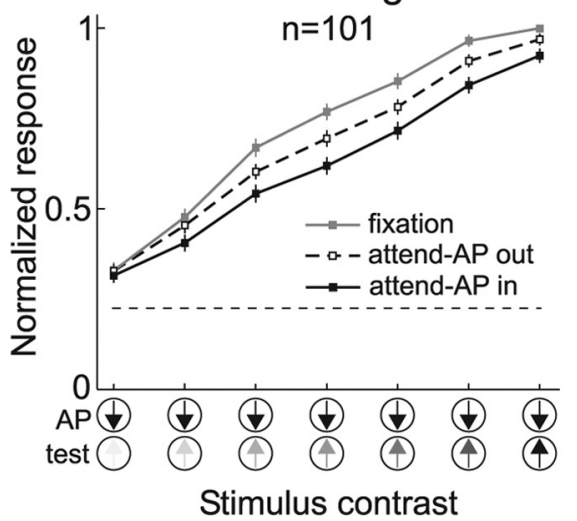

B

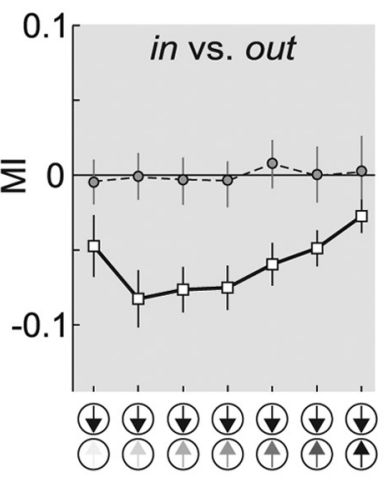

C

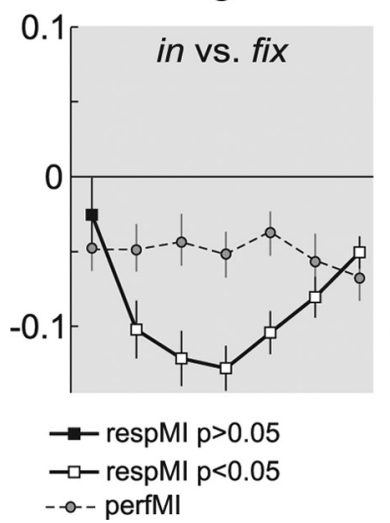

D

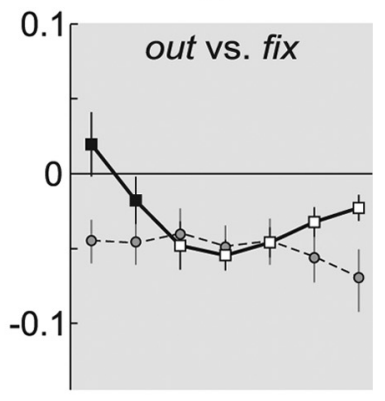

Figure 6. Attentional modulation in the contrast configuration. $\boldsymbol{A}$, Averaged normalized population responses $(n=101)$ for each stimulus pair of the contrast configuration, in the "fixation" (gray), "attend-AP in" (black), and "attend-AP out" (dashed) conditions. The horizontal line indicates spontaneous response without stimulus. Error bars, SEM. B-D, Averaged response MI (respMI, solid line and squares) and performance MI (perfMI, dashed line and circles) in the contrast configuration between the "attend-AP in" and "attend-AP out" (B) , "attend-AP in" and "fixation" ( $\boldsymbol{C}$ ), and "attend-AP out" and "fixation" conditions (D). The respMls were computed for each neuron and then pooled across units $(n=101)$. Similarly, the perfMls were computed for each recording session and then pooled across sessions ( $n=96$ ). Open squares denote respMls significantly different from zero and black squares denote nonsignificant ones. Error bars, SEM for the respMI and $95 \%$ confidence intervals for the perfMl.

sion during trials with intermediate contrast $(\mathrm{MI}=-0.128, p<$ $10^{-9}, t$ test). The difference between these two points (13\%) was highly significant ( $p<10^{-6}$, paired $t$ test). Relative to the previous comparison, these attentional effects are stronger, likely because they combine the effects of spatial and feature-based attention (i.e., directing attention from fixation to the antipreferred direction inside the RF). Although the animals' performance was higher during "fixation" compared to "attend-AP in" trials across all stimulus combinations (Fig. 6C, dashed line) (perfMI lower than zero, $p<10^{-6}$, sign test), these differences do not follow the shape of the differences in response quantified by the attentional MIs. The perfMI was similar across all contrast combinations ( $p>0.1$, Kruskal-Wallis ANOVA). Again, in this dataset, the differences in performance cannot account for the shape of the differences in attentional modulation (dashed line vs solid line).

We finally compared responses between the "fixation" and "attend-AP out" conditions (Fig. 6D). Here, the magnitude of the attentional modulation was smaller than in the previous comparison, but it followed a similar shape. The MIs were significantly different from zero across most contrast levels (white data points, $p<0.02$, $t$ test), and the pattern of larger differences for intermediate-contrast stimuli was also present. Responses to intermediate contrast were significantly suppressed by $10.5 \%$ $(\mathrm{MI}=-0.055)$ compared to $4.5 \%(\mathrm{MI}=-0.023)$ to the highestcontrast stimulus ( $p<0.01$, paired $t$ test). The perfMI did not follow the trend observed in the MIs, confirming that the pattern of attentional modulation was not due to differences in performance between conditions. Noticeably, attentional modulation in Figure $6 C$ was clearly larger than in Figure $6 D$, but the perfMIs were almost identical. This may reflect in the former case the combined effects of feature- and space-based attention, while in the latter case the isolated effect of feature-based attention.

In summary, these data show that spatial and feature-based attention and their combination modulate responses to intermediate-contrast stimuli more strongly than responses to low- and high-contrast stimuli. This demonstrates the contrast dependency of the modulation.

\section{Attentional modulation as a function of the test-pattern direction}

Our data in the direction configuration allowed us to further examine whether the magnitude of the attentional modulation changed as a function of the test-pattern motion direction in the absence of changes in stimulus contrast. We computed averaged population responses during trials of the direction configuration in the three behavioral conditions (Fig. 7A). Surprisingly, we found that relative to "fixation" (gray), responses in the "attend-AP in" (black) and "attend-AP out" (dashed line) conditions were suppressed for directions of the test pattern similar to the preferred (rightmost data points) but enhanced for directions progressively closer to the attended antipreferred direction (leftmost data points). This suppression/enhancement pattern was also consistent across the majority of the individual neurons (see cell example in Fig. $3 A$, right).

We quantitatively assessed the magnitude of these effects by determining for each neuron and direction combination the MIs between the "fixation" and the "attend-AP in" (Fig. 7C) or "attend-AP out" (Fig. 7D) conditions. In the first comparison the average MIs pooled across neurons became more positive as the direction of the test pattern approached the attended direction. "Attend-AP in" responses were modulated within a 23.5\% range, shifting from $10 \%$ response suppression (rightmost data point, $\mathrm{MI}=-0.051, p<10^{-6}, t$ test) to $13.5 \%$ enhancement (leftmost data point, $\mathrm{MI}=0.063, p<0.01, t$ test) relative to the "fixation" responses. The results for the comparison "fixation" versus "attend-AP out" (Fig. 7D) (which mainly isolates the effect of feature-based attention) were similar. Here, attentional effects shifted from $4.5 \%$ response suppression $(\mathrm{MI}=-0.023, p<0.02$, $t$ test) to $16 \%$ enhancement $(\mathrm{MI}=0.074, p<0.005, t$ test $)$. This shift in MIs from negative to positive in both comparisons, cannot be explained by variations in performance, which was higher in the "fixation" compared to the other two conditions for each stimulus combination (negative perfMI, dashed line, $p<10^{-9}$, sign test), but constant across all stimuli ( $p>0.1$, Kruskal-Wallis ANOVA).

One possible explanation for this effect is that in our task, feature-based attention (Martinez-Trujillo and Treue, 2004) dif- 

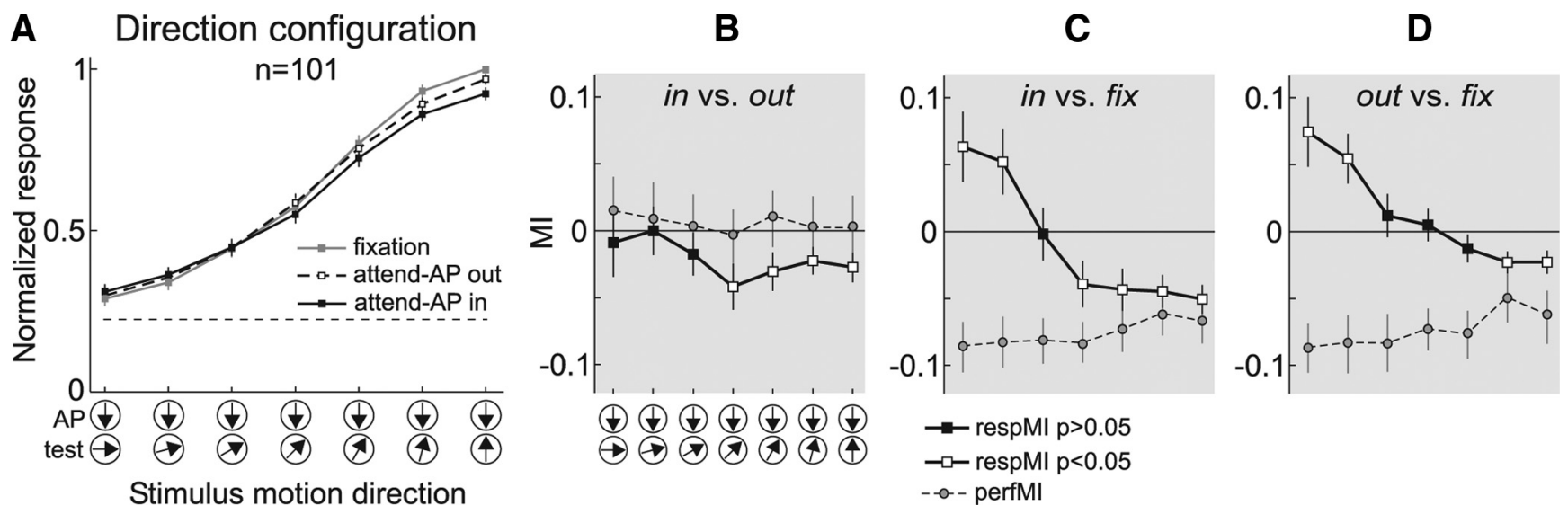

Figure 7. Attentional modulation in the direction configuration. $\boldsymbol{A}$, Averaged normalized population responses $(n=101)$ in the direction configuration. $\boldsymbol{B}-\boldsymbol{D}$, Averaged respMl and perfMI for the three comparisons. The same conventions are used as in Figure 6.

ferentially modulated the activation strength of two populations of direction-selective inputs carrying signals from each pattern into the recorded MT neuron. Several models have proposed this input modulation as a possible mechanism of attention (Reynolds et al., 1999; Ghose and Maunsell, 2008; Ghose, 2009; Reynolds and Heeger, 2009). When the animal allocates attention to the MT neuron's antipreferred direction (AP pattern) the strength of the inputs activated by that pattern (and therefore selective for that direction) would be enhanced. On the other hand, the strength of the inputs activated by the test pattern could change from a relatively strong suppression, when the activated inputs are selective for a direction $180^{\circ}$ away from the attended direction, to a progressively weaker suppression when the activated inputs are selective for directions progressively away from the preferred and closer to the attended antipreferred direction. When the total amount of enhancement of inputs activated by the AP pattern overcomes the progressively weaker suppression of inputs activated by the test pattern, the modulation switches sign, becoming predominantly a response enhancement relative to fixation.

Note that within this interpretation what defines the enhancement/suppression pattern due to feature-based attention is the relationship between the selectivity of the inputs and the attended direction. The same feature-based effects defined with respect to the MT neuron selectivity will show no changes in response since in our experiments the animals attended to the same motion direction across the different direction combinations (see Martinez-Trujillo and Treue, 2004). Also note that this effect of feature-based attention is defined relative to the "fixation" (neutral with respect to the attended feature) condition.

Although we cannot directly test this hypothesis, we could test at least one of its predictions. Removing the contribution of feature-based attention should abolish the shift in response modulation. We therefore compared responses between "attend-AP in" and "attend-AP out" trials, in which the modulation should mainly reflect the effect of spatial attention. We found that the shift in modulation disappeared (Fig. 7B). The response increase for directions of the test pattern more similar to the attended direction vanished (black data points), while a small response suppression remained for directions more dissimilar to the attended direction (white data points). However, across different direction pairs, there were no significant differences $(p>0.05$, one-way ANOVA). This effect was not due to changes in the animal performance, since performance was comparable in all trial types of both conditions (perfMI, dashed line, $p>0.8$, Kruskal-Wallis ANOVA). Overall, these data are compatible with the effect of feature-based attention on the strength of direction-selective inputs carrying signals from the AP and test pattern into the recorded neurons.

A question that arises when comparing the results of Figure 7 and Figure 4, $A, C$, and $E$, is why the attentional modulation as a function of motion direction was, in the latter case, almost negligible. The explanation is that in this analysis we pooled, within a given response level, responses to stimuli with different directions. As shown in Figure 7, $C$ and $D$, the modulation changed as a function of the test-pattern direction, thus, pooling responses corresponding to different directions would "cancel out" or "attenuate" attentional effects.

\section{Discussion}

We showed that, first, attentional modulation of similar response levels was systematically dependent on the visual stimulus. Second, attentional effects were stronger for intermediate-contrast stimuli. Third, the sign and magnitude of attentional modulation changed with the test-pattern motion direction, an effect attributable to feature-based attention.

\section{Attentional modulation as a function of response level}

Previous studies have reported attentional modulation of responses that are independent of stimulus orientation, direction, and contrast in MT and V4 neurons (McAdams and Maunsell, 1999; Treue and Martínez Trujillo, 1999; Williford and Maunsell, 2006), and a recent study has reported a contrast-independent modulation of V1 responses (Thiele et al., 2009). Others, however, have reported contrast-dependent modulation in areas MT and V4 (Reynolds et al., 2000; Martínez-Trujillo and Treue, 2002). Here, we found that attentional modulation in MT neurons was stronger for the contrast relative to the direction stimuli. Unlike previous studies, our approach produced a situation where distinct stimuli evoked the same response level. When controlling for the allocation of attention, this situation yields a clear prediction for the stimulus-independent modulation, i.e., a similar amount of response change for both stimulus configurations. Our data clearly argue against this prediction.

Furthermore, spatial, feature-based attention, and their combination produced similar effects, suggesting that the different types of attention share similar constraints. Differences between the contrast and direction data were also larger when combining 
spatial and feature-based attention than when each one acted alone, supporting previous reports of additive interactions between these types of attention (Treue and Martínez Trujillo, 1999; McAdams and Maunsell, 2000; Hayden and Gallant, 2009).

\section{Modulation as a function of stimulus contrast}

Our results in the contrast configuration agree with previous findings of a stronger attentional modulation for intermediatecontrast stimuli (Reynolds et al., 2000; Martínez-Trujillo and Treue, 2002); however, they apparently disagree with other reports of contrast-independent modulations (Williford and Maunsell, 2006; Thiele et al., 2009).

One may argue that differences in attentional effort associated with contrast changes may have confounded our results (Spitzer et al., 1988; Ghose and Maunsell, 2002; Boudreau et al., 2006), therefore explaining differences between studies. In our design, attentional effort was kept similar across both configurations by instructing the animals to always direct attention to a highcontrast stimulus (see performance data). It may be possible that when the test pattern had lower contrast the task was somewhat easier, and that although that was not reflected in the animals' performance, it may have influenced our results. At least three other findings discard this argument. First, for the lowest- and highest-contrast stimuli, where this hypothesis predicts the largest differences, the response modulation was similar, but it was in both cases different from the one for intermediate-contrast stimuli. Second, the comparisons "fixation" versus either "attend-AP in," or "attend-AP out," yielded considerable differences in modulation strength, however, their corresponding perfMIs were identical. Third, attentional effects were larger in the contrast compared to the direction configuration. The effect of task difficulty would have predicted the opposite result (i.e., larger modulation in the direction data where contrast was always high).

A more likely source of variability in results among studies is differences in task timing. The study reporting the largest variability in results across neurons has used the shortest stimulus presentation (94 ms) and analysis period (150 ms) (Williford and Maunsell, 2006). Another V4 study used a longer stimulus presentation ( $250 \mathrm{~ms}$ ) and analysis period ( $400 \mathrm{~ms}$ ) (Reynolds et al., 2000), obtaining a contrast-dependent modulation similarly as in our study (see also Martínez-Trujillo and Treue, 2002). This suggest that the dependency of the modulation on contrast becomes more evident for longer stimulus presentations and analysis periods. At least two different factors may cause this effect. First, because response latency in visual neurons increases when decreasing contrast (Reynolds and Desimone, 2003; Lee et al., 2007), integrating responses during early and short periods may underestimate firing rates and attentional effects associated to low-contrast stimuli. Second, transient stimuli may initially trigger exogenous attention, which if interacting with endogenous attention may produce different effects on responses during early periods. Interestingly, psychophysical experiments in humans have reported contrast-independent modulation of psychometric functions by exogenous attention, and contrast-dependent modulation by endogenous attention (Carrasco, 2006).

A number of studies have proposed quantitative models in which attention changes inputs' strength to an area normalization circuit (Reynolds et al., 1999; Raizada and Grossberg, 2003; Reynolds and Chelazzi, 2004; Ghose, 2009; Lee and Maunsell, 2009; Reynolds and Heeger, 2009). Our results are compatible with these models. On the other hand, they are not compatible with models in which a neuron's response function is scaled or shifted along the response axis by attention (Treue and Martínez
Trujillo, 1999; Williford and Maunsell, 2006; Boynton, 2009; Thiele et al., 2009).

An intriguing question is why the only existing study in V1 neurons reported a contrast-independent attentional modulation (Thiele et al., 2009). One possible explanation is that at the level of V1 neurons, attention uses a different mechanism than input strength modulation, wherein contrast increases do not constraint the modulation's magnitude. At least for featurebased attention this may make sense, since selectivity for some stimulus features such as motion direction arises in V1 (Peterson et al., 2004). Thus, an input modulation based on the attended feature (see next section) may not be possible. A target question for future studies is whether attentional modulation in thalamic lateral geniculate nucleus (McAlonan et al., 2008), and superior colliculus (Ignashchenkova et al., 2004) neurons, which are not direction selective, is contrast independent.

One may ask whether our results only apply to cases in which the effect of attention is associated with a decrease in firing rate (Martínez-Trujillo and Treue, 2002) rather than with an increase (Reynolds et al., 2000; Williford and Maunsell, 2006; Thiele et al., 2009). In our experimental design, these two processes likely occur in two different neuronal populations selective to opposite features (i.e., motion direction in MT, orientation in V4). We are inclined to believe that both process are constrained in contrast, because in the study of Reynolds et al. (1999), and in some of the neurons recorded by Williford and Maunsell (2006), increases in response by attention showed a similar contrast dependency as the response decreases shown in our study. However, whether the strength of the contrast-dependent attentional modulation between these two processes varies remains to be clarified.

\section{Modulation as a function of the test-pattern direction}

A surprising finding in our study was that in the direction configuration when animals switched attention between the fixation spot and the AP pattern inside or outside the RF, responses to the stimulus pair changed as a function of the test-pattern direction (Fig. 7). This result is different from the quasi-multiplicative modulation previously reported using a similar task (Treue and Martínez Trujillo, 1999). We believe there are two main reasons for these differences. First, that study sampled a limited number of test-pattern directions (every $30^{\circ}$ ), while here we sampled the direction space every $15^{\circ}$, providing a better resolution to detect differences in attentional modulation along the response function. Second, in the current study we may have included a sample of MT neurons more narrowly tuned for motion direction than in the previous study, since we actively searched for neurons that showed a similar decay in response within a $90^{\circ}$ range as with decreases in contrast. In the previous study, we recorded from any neuron that showed direction selectivity within a $360^{\circ}$ range, which may have resulted in a sample of more broadly tuned neurons where the direction-dependent modulation may become less evident.

We hypothesize that the test-pattern direction-dependent modulation may be caused by feature-based attention differentially modulating the strength of direction-selective inputs carrying signals from the AP and test pattern into the recorded neurons (Reynolds et al., 1999). These inputs may originate from area V1 direction-selective cells projecting toward the recorded MT neuron (Born and Bradley, 2005; Rust et al., 2006), whose responses could be modulated by attention (Motter, 1993; Roelfsema et al., 1998; Khayat et al., 2006). We did not directly test this hypothesis; however, two findings support it. First, the shift from suppression to enhancement was present when the animals 
switched attention between the fixation spot and the AP pattern outside the RF (feature-based attention). In the contrast configuration the shift was not present, indicating that it was not due to a bias introduced by low firing rates when the test-pattern direction deviated from the preferred direction; for low test-pattern contrasts, responses were also low. Second, the response shift disappeared when the animal switched attention from the AP pattern outside the RF to the one inside (spatial attention).

In general, our results support models in which spatial and feature-based attention modulate the strength of directionselective inputs from attended and unattended stimuli into the MT normalization circuit. Clarifying whether this modulation occurs at the level of population of neurons in areas projecting toward MT, or at the level of synaptic inputs into MT neurons, or both, remains as a challenge for future studies.

\section{References}

Born RT, Bradley DC (2005) Structure and function of visual area MT. Annu Rev Neurosci 28:157-189.

Boudreau CE, Williford TH, Maunsell JHR (2006) Effects of task difficulty and target likelihood in area V4 of macaque monkeys. J Neurophysiol 96:2377-2387.

Boynton GM (2009) A framework for describing the effects of attention on visual responses. Vision Res 49:1129-1143.

Buracas GT, Boynton GM (2007) The effect of spatial attention on contrast response functions in human visual cortex. J Neurosci 27:93-97.

Busse L, Katzner S, Treue S (2008) Temporal dynamics of neuronal modulation during exogenous and endogenous shifts of visual attention in macaque area MT. Proc Natl Acad Sci U S A 105:16380-16385.

Carrasco M (2006) Covert attention increases contrast sensitivity: psychophysical, neurophysiological and neuroimaging studies. Prog Brain Res 154:33-70.

Desimone R, Duncan J (1995) Neural mechanisms of selective visual attention. Annu Rev Neurosci 18:193-222.

Ekstrom LB, Roelfsema PR, Arsenault JT, Kolster H, Vanduffel W (2009) Modulation of the contrast response function by electrical microstimulation of the macaque frontal eye field. J Neurosci 29:10683-10694.

Ghose GM (2009) Attentional modulation of visual responses by flexible input gain. J Neurophysiol 101:2089-2106.

Ghose GM, Maunsell JHR (2002) Attentional modulation in visual cortex depends on task timing. Nature 419:616-620.

Ghose GM, Maunsell JHR (2008) Spatial summation can explain the attentional modulation of neuronal responses to multiple stimuli in area V4. J Neurosci 28:5115-5126.

Hayden BY, Gallant JL (2009) Combined effects of spatial and feature-based attention on responses of V4 neurons. Vision Res 49:1182-1187.

Ignashchenkova A, Dicke PW, Haarmeier T, Thier P (2004) Neuronspecific contribution of the superior colliculus to overt and covert shifts of attention. Nat Neurosci 7:56-64

Khayat PS, Spekreijse H, Roelfsema PR (2006) Attention lights up new object representations before the old ones fade away. J Neurosci 26:138-142.

Lee J, Maunsell JH (2009) A normalization model of attentional modulation of single unit responses. PLoS One 4:e4651.

Lee J, Williford T, Maunsell JH (2007) Spatial attention and the latency of neuronal responses in macaque area V4. J Neurosci 27:9632-9637.
Li X, Lu ZL, Tjan BS, Dosher BA, Chu W (2008) Blood oxygenation leveldependent contrast response functions identify mechanisms of covert attention in early visual areas. Proc Natl Acad Sci U S A 105:6202-6207.

Martínez-Trujillo J, Treue S (2002) Attentional modulation strength in cortical area MT depends on stimulus contrast. Neuron 35:365-370.

Martinez-Trujillo JC, Treue S (2004) Feature-based attention increases the selectivity of population responses in primate visual cortex. Curr Biol 14:744-751.

Maunsell JH, Treue S (2006) Feature-based attention in visual cortex. Trends Neurosci 29:317-322.

McAdams CJ, Maunsell JH (1999) Effects of attention on orientationtuning functions of single neurons in macaque cortical area V4. J Neurosci 19:431-441.

McAdams CJ, Maunsell JH (2000) Attention to both space and feature modulates neuronal responses in macaque area V4. J Neurophysiol 83:1751-1755.

McAlonan K, Cavanaugh J, Wurtz RH (2008) Guarding the gateway to cortex with attention in visual thalamus. Nature 456:391-394.

Motter BC (1993) Focal attention produces spatially selective processing in visual cortical areas V1, V2, and V4 in the presence of competing stimuli. J Neurophysiol 70:909-919.

Moulden B, Kingdom F, Gatley LF (1990) The standard deviation of luminance as a metric for contrast in random-dot images. Perception 19:79-101.

Murray SO (2008) The effects of spatial attention in early human visual cortex are stimulus independent. J Vis 8:2.1-2.11.

Peterson MR, Li B, Freeman RD (2004) The derivation of direction selectivity in striate cortex. J Neurosci 24:3583-3591.

Raizada RD, Grossberg S (2003) Towards a theory of the laminar architecture of cerebral cortex: computational clues from the visual system. Cereb Cortex 13:100-113.

Reynolds JH, Chelazzi L (2004) Attentional modulation of visual processing. Annu Rev Neurosci 27:611-647.

Reynolds JH, Desimone R (2003) Interacting roles of attention and visual salience in V4. Neuron 37:853-863.

Reynolds JH, Heeger DJ (2009) The normalization model of attention. Neuron 61:168-185.

Reynolds JH, Chelazzi L, Desimone R (1999) Competitive mechanisms subserve attention in macaque areas V2 and V4. J Neurosci 19:1736-1753.

Reynolds JH, Pasternak T, Desimone R (2000) Attention increases sensitivity of V4 neurons. Neuron 26:703-714.

Roelfsema PR, Lamme VAF, Spekreijse H (1998) Object-based attention in the primary visual cortex of the macaque monkey. Nature 395:376-381.

Rust NC, Mante V, Simoncelli EP, Movshon JA (2006) How MT cells analyze the motion of visual patterns. Nat Neurosci 9:1421-1431.

Spitzer H, Desimone R, Moran J (1988) Increased attention enhances both behavioral and neuronal performance. Science 240:338-340.

Thiele A, Pooresmaeili A, Delicato LS, Herrero JL, Roelfsema PR (2009) Additive effects of attention and stimulus contrast in primary visual cortex. Cereb Cortex 19:2970-2981.

Treue S (2001) Neural correlates of attention in primate visual cortex. Trends Neurosci 24:295-300.

Treue S, Martínez Trujillo JC (1999) Feature-based attention influences motion processing gain in macaque visual cortex. Nature 399:575-579.

Williford T, Maunsell JHR (2006) Effects of spatial attention on contrast response functions in macaque area V4. J Neurophysiol 96:40-54. 